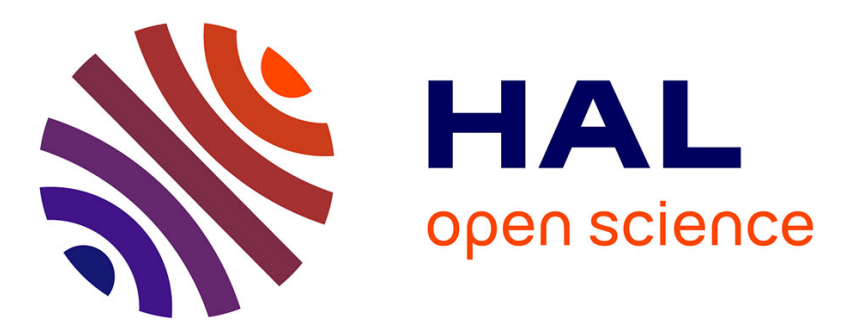

\title{
Foam Drainage Control Using Thermocapillary Stress in a Two-Dimensional Microchamber
}

Vincent Miralles, Bertrand Selva, Isabelle Cantat, Marie-Caroline Jullien

\section{To cite this version:}

Vincent Miralles, Bertrand Selva, Isabelle Cantat, Marie-Caroline Jullien. Foam Drainage Control Using Thermocapillary Stress in a Two-Dimensional Microchamber. Physical Review Letters, 2014, 112, pp.238302. 10.1103/PhysRevLett.112.238302 . hal-01006595

\section{HAL Id: hal-01006595 https://hal.science/hal-01006595}

Submitted on 16 Jun 2014

HAL is a multi-disciplinary open access archive for the deposit and dissemination of scientific research documents, whether they are published or not. The documents may come from teaching and research institutions in France or abroad, or from public or private research centers.
L'archive ouverte pluridisciplinaire HAL, est destinée au dépôt et à la diffusion de documents scientifiques de niveau recherche, publiés ou non, émanant des établissements d'enseignement et de recherche français ou étrangers, des laboratoires publics ou privés. 


\title{
Foam drainage control using thermocapillary stress in a two-dimensional microchamber.
}

\author{
V. Miralles ${ }^{1}$, B. Selva ${ }^{2}$, I. Cantat ${ }^{3}$, M.-C. Jullien ${ }^{1}$ \\ ${ }^{1}$ MMN, CNRS, ESPCI Paris-Tech, 10 rue Vauquelin, F-75005 Paris, France. \\ ${ }^{2}$ LOF, unité mixte Solvay-CNRS-Bordeaux 1, 178 avenue du Docteur Schweitzer, F-33608 Pessac cedex, France. \\ ${ }^{3}$ IPR, (UMR CNRS 6251), Université de Rennes 1, 35000 Rennes, France.
}

\begin{abstract}
We investigate the drainage of a 2D microfoam in a vertical Hele-Shaw cell, and show that the Marangoni stress at the air-water interface generated by a constant temperature gradient applied in situ can be tuned to control the drainage. The temperature gradient is applied in such a way that thermocapillarity and gravity have an antagonist effect. We characterize the drainage over time by measuring the liquid volume fraction in the cell and find that thermocapillarity can overcome the effect of gravity, effectively draining the foam towards the top of the cell, or exactly compensate it, maintaining the liquid fraction at its initial value over at least $60 \mathrm{~s}$. We quantify these results by solving the mass balance in the cell, and provide insight on the interplay between gravity, thermocapillarity and capillary pressure governing the drainage dynamics.
\end{abstract}

The development of controlled materials containing gas inclusions covers a large variety of applications such as scaffolds for tissue engineering [1, 2], enhanced oil recovery [3, 4], optics [5, 6] and phononic crystals [7] to cite a few. Microfluidics gives access to structures unachievable at the macroscopic scale $[8,9]$ and recent studies show that a major difficulty in manufacturing such discrete materials is to control the foam stabilization [10-12], or destabilization [13, 14] over time, involving three phenomena : i) drainage due to gravity, ii) diffusion of gas through the liquid films, and iii) coalescence of neighboring bubbles [15]. Although microfluidics has proven to be an efficient technology to generate monodisperse bubbles [16], gravity drainage still affects the organization of large number of these bubbles in vertical 2D or 3D matrices - even at such small scales. Recent attempts at foam drainage control have involved introducing activable materials in the carrier phase, such as magnetic particles or photo-sensitive surfactants [12-14]. In this paper, we investigate a new way to control foam drainage using thermocapillary Marangoni effects, which refers to a mass transfer along an interface between two fluids due to a temperature-induced surface tension gradient. We demonstrate that the application of a constant temperature gradient to a 2D microfoam can stop or reverse the natural drainage due to gravity. A mass transfer balance using scaling arguments allows us to provide physical insight on our quantitative experiments. We believe this study in a $2 \mathrm{D}$ model experiment to be a useful building block towards foam drainage control in more complex situations.

Our experimental chamber is a Polydimethylsiloxane (PDMS) Hele-Shaw cell fabricated using soft-lithography [17], of dimensions $\mathrm{L} \times \mathrm{w} \times \mathrm{e}=2000 \times 1500 \times 54.5 \mu \mathrm{m}^{3}$ respectively in the $x, y$ and $z$ directions, Fig.1. When the cell dimensions or the cell orientation are modified, it is specified in the text. Bubbles are generated at a flow-focusing junction $[16,18,19]$ into a capillary tube that divides into five intermediate microchannels, allowing for even spreading of the bubbles in the Hele Shaw cell. The total volume of the microchannels (inlet plus outlet) is $16 \%$ of the cell volume. Once the cell is entirely filled with bubbles, the capillary tube is disconnected. The solution is free to exit the cell from the bottom or from the top, depending on the force driving the drainage. In the following we call outlet the intermediate microchannels towards which the liquid drains, and inlet the opposite ones. The number of bubbles inside the cell remains constant throughout each experiment. In each experiment, the initial liquid volume fraction is $\phi_{0} \sim 14 \%$ and the polydispersity of the foam (standard deviation over the mean value of the bubble radius distribution) is typically lower than $8 \%$. The foaming solution consists of an anionic surfactant solution (SDS, Sigma-Aldrich, at $9 \mathrm{mM}$ ) mixed with glycerol (5.68 wt $\%$, Aldrich) and titanium dioxide (5.68 wt\%, Sachtleben) in deionized water (Millipore). Surfactant guarantee the presence of a lubrication film between the bubbles and the walls. The surface tension of the solution is measured with a Wilhelmy plate and found to be $\gamma=30.8 \pm 0.1 \mathrm{mN} \cdot \mathrm{m}^{-1}$ at $20^{\circ} \mathrm{C}$, while the dependency on temperature is $\partial_{T} \gamma=-2.1 \pm 0.110^{-4}$ $\mathrm{N} \cdot \mathrm{m}^{-1} \cdot \mathrm{K}^{-1}$. The dynamic viscosity and density of the solution are respectively $\eta=1.1510^{-3}$ Pa.s and $\rho=10^{3} \mathrm{~kg} . \mathrm{m}^{-3}$. The forcing parameter is a well-controlled constant tempera-

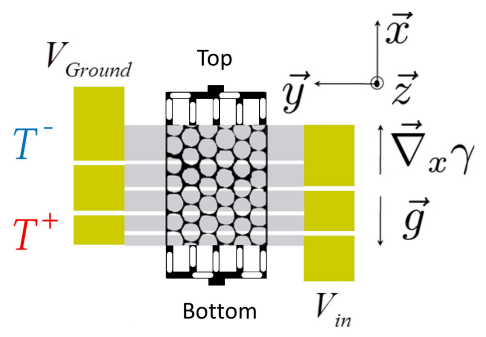

FIG. 1: Sketch of the experimental setup. The chromium heating resistors (grey stripes) are connected to the voltage source via gold wires (golden stripes). The Hele-Shaw cell is sealed above the heating resistors. The liquid (resp. air) is represented in black (resp. white).

ture gradient oriented along the vertical $x$-axis [20, 21]. We checked in [22] that it is not significantly altered by the flow, so that $\mathrm{d}_{x} \gamma=\left(\partial_{T} \gamma\right)\left(\partial_{x} T\right)$ is also constant. We apply a negative $\partial_{x} T$ in our experiments, so that the surface tension gradient is positive and induces a Marangoni flow towards the top of the cell, i.e. in the opposite direction to gravity. In the following, we denote by $\partial_{x} T$ the absolute value of the temper- 
ature gradient (for the sake of simplicity).

The experimental cell is placed on a stereomicroscope (Leica MZ FLIII) fitted with a white light source (Volpi Intralux 5100) in reflection configuration. The foam evolution is recorded with a CMOS camera (PixeLink PL-B781) at a 2 $\mathrm{Hz}$ acquisition frame rate. The presence of titanium dioxide makes the solution very diffusive (the characteristic optical transport length is slightly larger than the wetting film thickness along the lateral walls) and leads to high contrast images where the thin films appear black while the menisci, called pseudo-Plateau borders (pPB), appear white (see Fig.2c). This visualization method ensures that the pictures are not distorted by refraction or reflection on the interfaces [23].
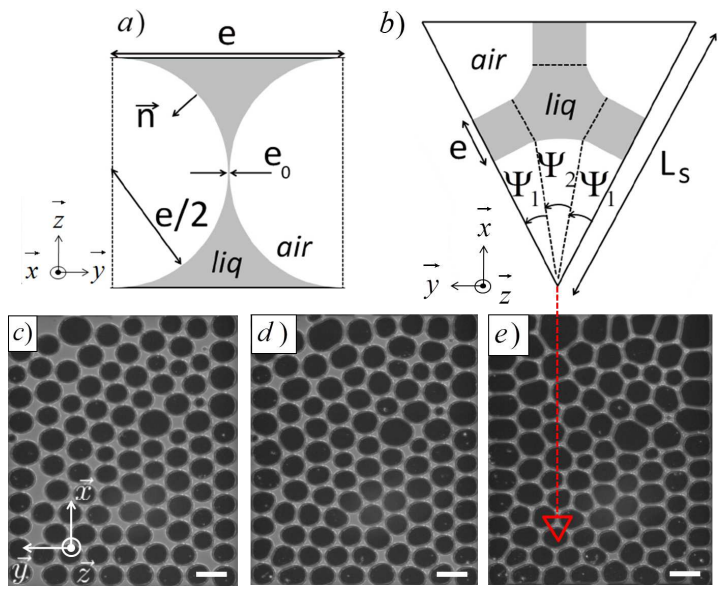

FIG. 2: (a) Cross-sectional view of a pseudo-Plateau border (pPB). Top views: (b) Parameters used to measure the liquid fraction $\phi$ over time; (c), (d) and (e) time evolution of a foam of initial liquid volume fraction $\phi_{0} \sim 14 \%$, at times $\mathrm{t}=0, \mathrm{t}=2 \mathrm{~s}$ and $\mathrm{t}=15 \mathrm{~s}$ for $\partial_{x} T=7.0$ $\mathrm{K} . \mathrm{mm}^{-1}$. Scale bars represent $200 \mu \mathrm{m}$.

Snapshots of the typical time evolution of the foam structure during an experiment are represented in Fig.2,c.d.e. In our confined geometry, the foam structure is highly constrained, and the whole 3D foam structure can be deduced from these snapshots [24, 25]. At an early stage (first regime), the foam is wet and the bubbles adopt a pancake shape of radius $R$, Fig 2c. The mean curvature of their lateral interface, which is not in contact with the plates, is $\kappa=2 / e+1 / R \sim 2 / e$ for large bubbles. Then, the bubbles come into contact as the liquid phase is drained out of the cell: Fig.2d shows the onset of a second regime. As the liquid fraction decreases, the contact point between bubbles is stretched and the bubbles adopt a polygonal shape, Fig.2e. The bubbles are separated by the $\mathrm{pPB}$, which meet at threefold vertices. The contact area between two bubbles in this regime is approximately a line along which the pPB in contact with both plates meet, Fig.2a. The interface curvature thus remains close to $2 / e$, which is the curvature of the $\mathrm{pPB}$ section, while the width of the $\mathrm{pPB}$ is equal to $e$. As $\phi$ decreases, the curvature of the vertices in the $(x y)$ plane, $1 / R_{v}$, slightly increases towards $1 / R_{v} \sim 2 / e$. In general, this point would correspond to the onset of a third regime, in which thin films form between the bubbles as the liquid continues to be drained $[24,25]$. We never enter this third regime and observe that the foam reaches a stationary state in which the curvature of the vertices is constant, and drainage stops. In this letter we focus on the first $60 \mathrm{~s}$ of the experiments where coarsening can be neglected. In what follows, we also discard the first few snapshots corresponding to the first regime during which the bubbles come into contact. We quantify the drainage dynamics in the second regime.

The foam exhibits no drainage front. Statistics over subregions of the cell (centered at different positions along $x$, containing about 10 bubbles) show that the liquid fraction determined with equation (1) is uniform with a precision $\Delta \phi \sim 10^{-2}$ given by the pixel size. We show at the end of this Letter that this is chiefly due to capillary pressure in the foam. In the following, $\phi$ accounts for the mean liquid fraction throughout the cell. To calculate $\phi$, we first measure the fraction of the plate in contact with the $\mathrm{pPB}, \phi^{2 D}$, obtained from the ratio of white to black pixels in the observation plane. We take advantage of the constant width of the pPB (equal to $e$ ) to set the threshold in the image processing (contact point between two adjacent bubbles, Fig.2d). The relative size of the edges and the vertices is quantified by the angles $\Psi_{1}$ and $\Psi_{2}$ defined Fig.2b. These angles are calculated from the number of bubbles per unit area, the measured $\phi^{2 D}$, and the assumption of a regular hexagonal foam. We assume then that the liquid-gas interface in the vertex is part of a torus, with one radius of curvature equal to $e / 2$, and the other one deduced from $\Psi_{2}$. Hence the liquid fraction $\phi$ writes:

$$
\begin{aligned}
\phi= & 1-\sqrt{3}\left(\tan \left(\Psi_{1}\right)+\frac{\Psi_{2}}{2 \cos \left(\Psi_{1}\right)^{2}}\right) \\
& \times\left(1-\left(\frac{e}{L_{S}}\right)\left(2-\frac{\pi}{2}\right)+\left(\frac{e}{L_{S}}\right)^{2}\left(\frac{5}{3}-\frac{\pi}{2}\right)\right)
\end{aligned}
$$

The evolution of the liquid fraction over time is plotted in Fig.3. When subjected to gravity only, the drainage occurs towards the bottom outlet, and $\phi$ decays until it reaches a plateau at $\phi \sim 4.5 \%$. For a precise value of the temperature gradient, $\partial_{x} T=3.1 \mathrm{~K} . \mathrm{mm}^{-1}$, the Marangoni contribution exactly balances the effect of gravity on drainage: the liquid fraction is maintained constant at its initial value. Upon increase of the temperature gradient, the drainage direction is reversed: the evolution of $\phi$ is similar to the pure gravity case, but the liquid is drained towards the top of the cell. The drainage can be quantified by solving the liquid transport in the foam, using scaling arguments. As the liquid fraction is uniform at each time step within experimental accuracy, the dynamics of the system can be predicted by a global mass balance in the cell. To perform such a mass balance, the flows at the inlet and outlet have to be determined. During the first regime, the bubbles in the inlet channels come into contact as the liquid drains out through the cell. Hence, we assume that there is no liquid reservoir -and so zero flux- at the inlet. At the outlet, as the bubbles are in direct contact with the liquid exiting the cell, we assume that the capillary pressure gradient tends to zero (no 


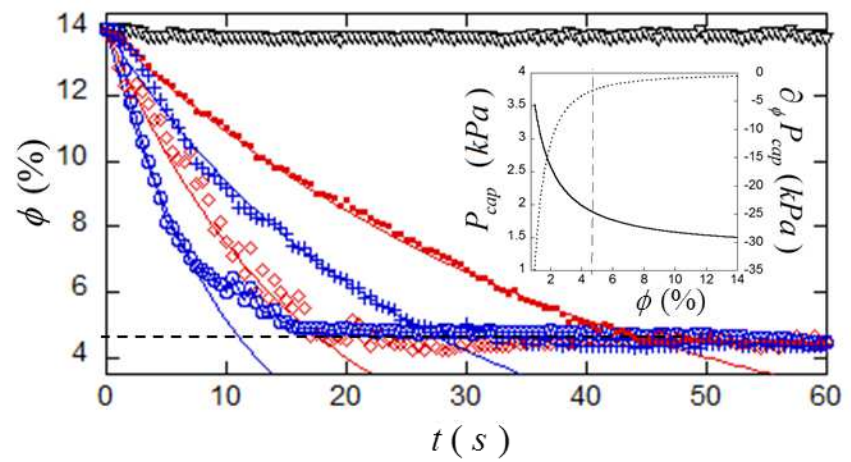

FIG. 3: Time evolution of the liquid fraction $\phi$ (cell placed vertically) for $\partial_{x} T=0(\diamond), 2.1(\bullet), 3.1(\nabla), 5.5(+)$ and $7.0(\circ){\mathrm{K} . m^{-1}}^{-}$. Solid lines show the best exponential fit for each dataset. The dashed line shows the saturation at $\phi \sim 4.5 \%$. Each datapoint, at a given time, is obtained by taking the mean value over five experiments. Error bars lie within $10 \%$ for all the experimental curves, and are not represented for a better clearness. Inset: capillary pressure drop in the foam $\left(P_{c a p}\right.$ - straight line $)$ and its derivative with respect to $\phi\left(\partial_{\phi} P_{c a p}\right.$ - dotted line), as a function of $\phi$ [25]. Note that the blue (resp. red) curves correspond to a drainage towards the cold (resp. hot) side of the cell.

capillary flow). Lastly, we presume that the liquid transport is dominant in the pPB. A simple dimensional argument leads to the following expressions for the thermocapillary and gravity contributions to the velocity of the liquid phase: $\bar{v}_{x}^{t h}=\alpha \mathrm{d}_{\mathrm{x}} \gamma e / \eta$ and $\bar{v}_{x}^{g}=-\beta e^{2} \rho g / \eta$, where $\bar{v}_{x}$ stands for the projection along $x$ of the average velocity in the liquid phase, $\alpha$ and $\beta$ are positive dimensionless parameters [26]. The thermocapillary and gravity fluxes across a section $(y z)$ of the cell are given by $Q^{\text {th }}=\bar{v}_{x}^{\text {th }} e w \phi$ and $Q^{g}=\bar{v}_{x}^{g} e w \phi$. The mass conservation equation in the cell thus reads $\mathrm{d}(e w L \phi) / \mathrm{dt}= \pm\left(Q^{t h}+Q^{g}\right)$, where the sign $+/-$ corresponds to fluid exiting respectively at the bottom (i.e. $\left.\left|\bar{v}_{x}^{t h}\right|<\left|\bar{v}_{x}^{g}\right|\right)$ or top of the cell $\left(\left|\bar{v}_{x}^{t h}\right|>\left|\bar{v}_{x}^{g}\right|\right)$. In both cases, we get:

$$
\ln \left(\frac{\phi}{\phi_{0}}\right)=-\left|\frac{1}{t_{d}^{t h}}-\frac{1}{t_{d}^{g}}\right| t=-\frac{t}{t_{d}}
$$

where $t_{d}^{t h}=\eta L /[\alpha e(\mathrm{~d} \gamma / \mathrm{d} x)]$ and $t_{d}^{g}=\eta L /\left[\beta \rho g e^{2}\right]$ are characteristic drainage times due to thermocapillarity and gravity respectively.

Fitting our data points with an exponential decay before the saturation, we measure $t_{d}=t_{d}^{g}=18.2 \pm 0.2 \mathrm{~s}$ for gravity only and $t_{d} \in\{49.3 \pm 0.5 ; 26.9 \pm 0.3 ; 16.2 \pm 0.2\} \mathrm{s}$ for $\partial_{x} T \epsilon$ $\{2.1 ; 5.5 ; 7.0\} \mathrm{K} . \mathrm{mm}^{-1}$ respectively, Fig.3. We deduce $\beta=$ $4.7 \pm 0.210^{-3}$ from $t_{d}^{g}$. In order to make an independent measurement of $\alpha$, the cell is placed horizontally to eliminate the influence of gravity, inset Fig.4. When plotting $\ln \left(\phi / \phi_{0}\right)$ as a function of $t / t_{d}$, all the curves collapse on the same straight line of slope -1 for $\alpha=3.7 \pm 0.110^{-3}$, Fig.4. Reinjecting these independently-measured values of $\alpha$ and $\beta$ in $t_{d}$, we find respectively $t_{d} \in\{49.3 \pm 0.5 ; 26.9 \pm 0.3 ; 16.2 \pm 0.2\} \mathrm{s}$ for $\partial_{x} T \in\{2.1 ; 5.5 ; 7.0\} \mathrm{K} . \mathrm{mm}^{-1}$ for experiments where both gravity and thermocapillarity intervene, in good agree- ment with our best fits. The balance between thermocapillarity and gravity is given by $t_{d}^{g}=t_{d}^{t h}$, which yields $\partial_{x} T=3.1$ K. $\mathrm{mm}^{-1}$. This shows that our minimal mass balance argument is sufficient to shed physical insight on the drainage dynamics at short times (see Suppl. Mat. for negligible additional effects). In particular, this validates the assumption of negligible capillary flux at the outlet and negligible total flux at the inlet.

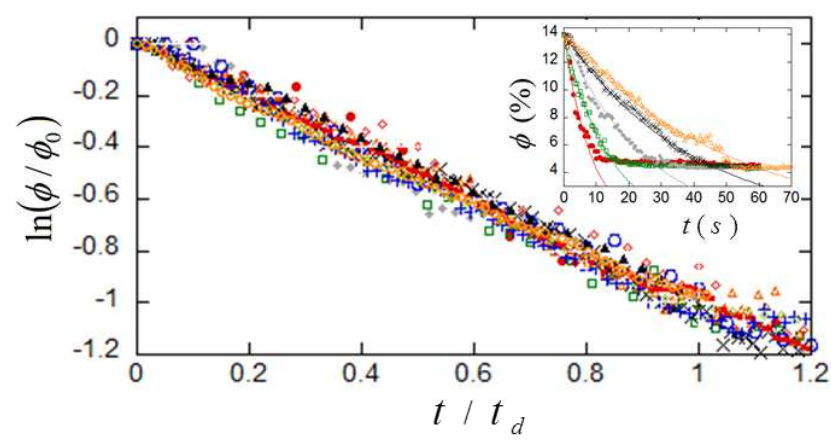

FIG. 4: $\ln \left(\phi / \phi_{0}\right)$ as a function of $t / t_{d}$. Data correspond to $(\diamond)$, $(\bullet),(+)$ and (o) presented in Fig.3 (cell placed vertically), and to six experiments performed with the cell placed horizontally (see inset):

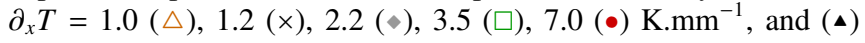
corresponds to an experiment with $\mathrm{C}_{12} \mathrm{TAB}$ surfactant at $\partial_{x} T=7.0$ $\mathrm{K} . \mathrm{mm}^{-1}$. Data $(\circ)$ and $(\diamond)$ correspond to a cell $2500 \times 1500 \times 32 \mu \mathrm{m}^{3}$ placed horizontally, respectively for $\partial_{x} T=3.5$ and $7.0 \mathrm{~K} . \mathrm{mm}^{-1}$. Inset: time evolution of $\phi$ for the five experiments $\partial_{x} T=1.0(\triangle), 1.2$ $(\times), 2.2(\bullet), 3.5(\square), 7.0(\bullet) \mathrm{K} \cdot \mathrm{mm}^{-1}$ (cell placed horizontally). Each datapoint, at a given time, is obtained by taking the mean value over five experiments. Solid lines show the best exponential fit for each dataset.

In order to check the robustness of the model, we performed complementary experiments with another soluble surfactant, and with cells of different thicknesses and lengths (see Suppl. Mat.), and lead to values of $\alpha$ and $\beta$ consistent with the expected model.

The saturation value is independent of the forcing parameter and is related to the emergence of a non negligible capillary pumping at the outlet, Fig.3 and inset Fig.4. More precisely, the capillary flux obeys a similar law to the gravity flux and writes $Q^{c a p}=(e w \phi)\left(\beta e^{2} / \eta\right) \mathrm{d}_{x} P, \mathrm{~d}_{x} \mathrm{P}$ being the driving force. Its expression is thus. The dependence of $P$ with $\phi$ can be determined using eq.22 of [25], see inset Fig.3. $\partial_{\phi} P$ remains of the order of a few $\mathrm{kPa}$ for $\phi$ larger than the saturation value and then diverges rapidly for smaller values. This transition below $\phi \sim 4.5 \%$ corresponds to the appearance of thin films separating the bubbles (third regime). This regime is not observed, as neither gravity nor thermocapillarity are strong enough to overcome the capillary pressure: instead, $\phi$ saturates at this limit value.

The influence of the capillary pressure is subtle. We can show that during the drainage process, the capillary flux in the cell is small but non-zero far from the outlet, and ensures the liquid fraction homogeneity. A local mass balance imposes $e w \partial \phi(x, t) / \partial t=\partial\left(Q^{g}(x)+Q^{t h}(x)+Q^{c a p}(x)\right) / \partial x$. We 
denote $\delta \phi(x, t)$ the small spatial variations of the liquid fraction relatively to its mean value in the cell $\phi(t)$. Since we measure experimentally $\delta \phi / \phi<0.1$, we can assume $Q^{g}, Q^{t h}$ and $\phi$ to be independent of $x$. In contrast, for $\partial_{\phi} P$ of the order of $1 \mathrm{kPa}$, as observed during the drainage phase (inset Fig.3), we get $\left(\partial_{\phi} P\right)\left(\partial_{x} \phi\right) \sim 10^{6} \delta \phi$ Pa.m $^{-1}$ over a typical length scale of order $1 \mathrm{~mm}$. Hence the capillary pressure gradient is non negligible compared to the hydrostatic pressure gradient, $\rho g \sim 10^{4} \mathrm{~Pa} . \mathrm{m}^{-1}$, even for $\delta \phi$ as small as $10^{-2}: \delta \phi$ can be neglected in all terms excepted $Q^{\text {cap }}(x)$ in the local mass balance. An integration from $x$ to $L$ yields $Q^{\text {cap }}(x)=(1-x / L)\left(Q^{g}(L)+Q^{\text {th }}(L)\right)$. Dealing with the pure gravity situation, we can predict $\partial \delta \phi / \partial x=(1-x / L) \rho g / \partial_{\phi} P$ in the cell, which leads to $\delta \phi \sim 6.10^{-3}$, a value well below our experimental uncertainty. A variation $\delta \phi / \phi$ of $10 \%$ is predicted for $L \sim 3.5 \mathrm{~mm}$, above which some capillary corrections need to be introduced in the model. In other words, though the capillary pressure is negligible at the outlet where the foam is in contact with the solution, it is high enough in the core of the cell to redistribute the liquid towards the inlet and to avoid the formation of a drainage front. As more liquid exits the cell, the capillary pressure gradient becomes of the same order as gravity or thermocapillarity even at the outlet, thus stopping the drainage.

To conclude, the main result of this work is that applying a thermocapillary Marangoni stress at the air-water interface is an effective way to control foam drainage in a microscale 2D geometry. More precisely, thermocapillarity can accurately counterbalance the effect of gravity, or overcome it, draining the liquid in the other direction. We rationalize our approach by writing down the mass conservation in the cell, which sheds light on the physics at play by defining characteristic drainage times. We wish to emphasize that this work can be used as an elementary step in a bottom-up approach towards drainage control in more complex systems and can pave the way to controlled discrete materials. First, the 2D Hele-Shaw cell and microscale of the experiments allow for full knowledge of the foam geometry, and therefore liquid fraction and drainage dynamics. Secondly, our experimental results can be accounted for using a purely hydrodynamical approach, contrary to previous studies involving surfactants with a more complex rheology [27, 28]. This study can be refined or extended to polydisperse 2D, 3D, or macroscopic foams, with non-constant temperature gradients and/or surface elasticity: we believe it to be of both basic and practical interest to many fields.

This work has been patented under the reference 26877FR, and supported by CNRS, DGA, IPGG (Equipex ANR-10EQPX-34), ESPCI and ANR under the contract 13-BS090011-01. We acknowledge valuable discussions with Benjamin Dollet, Michael Schindler, Thomas Salez and Elie Raphael.
[2] C. Colosi, M. Costantini, A. Barbetta, R. Pecci, R. Bedini and M. Dentini, Langmuir, 29, 82, 2013.

[3] R. Farajzadeh, A. Andrianov, R. Krastev, G.J. Hirasaki, W.R. Rossen, Advances in Colloid and Interface Science 183-184, 1-13, 2012.

[4] R. Liontas, K. Ma, G. J. Hirasaki and S. L. Biswal, Soft Matter 9, 10971, 2013.

[5] M. Hashimoto, B. Mayers, P. Garstecki and G. M. Whitesides, Small 2, 12921298, 2006

[6] F. Malloggi, N. Pannacci, R. Attia, F. Monti, P. Mary, H. Willaime, P. Tabeling, B. Cabane and P. Poncet, Langmuir 26, 2369, 2010.

[7] V. Leroy, A. Bretagne, M. Fink, H. Willaime, P. Tabeling, A. Tourin. Appl. Phys. Lett., 95, 171904, 2009.

[8] D. Weaire and W. Drenckhan, Adv. Colloid Interface Sci. 137, 20, 2008.

[9] D.Weaire, S. T. Tobin, A. J. Meagher and S. Hutzler, Foam Engineering, John Wiley \& Sons, Ltd, , p. 526, 2012.

[10] O. Bonhomme, O. Liot, A.-L. Biance and L. Bocquet. Phys. Rev. Lett., 110, 054502, 2013.

[11] A.-L Fameau, A. Saint-Jalmes, F. Cousin, B. Houinsou Houssou, B. Novales, L. Navailles, F. Nallet, C. Gaillard, F. Boué, and J.-P. Douliez. Angew. Chem., 50, 8264-8269, 2011.

[12] D. E. Moulton and J. A. Pelesko. Phys. Rev. E., 81, 046320, 2010.

[13] E. Chevallier, C. Monteux, F. Lequeux and C. Tribet. Langmuir, 28, 2308-2312, 2012.

[14] E. Chevallier, A. Mamane, H.A. Stone, C. Tribet, F. Lequeux and C. Monteux. Soft Matter, 7, 7866, 2011.

[15] D. Weaire and S. Hutzler. Oxford University Press, 1999, ISBN: 0198505515.

[16] P. Garstecki, I. Gitlin, W. DiLuzio and G.M. Whitesides. Appl. Phys. Lett., 85, 13, 2004.

[17] Y. Xia Y. and G.M. Whitesides. Angew. Chem. Int. Ed., 37, 550-575, 1998.

[18] B. Dollet, W. van Hoeve, J.-P. Raven, P. Marmottant and M. Versluis. Phys. Rev. Lett., 100, 034504, 2008.

[19] A. M. Ganan-Calvo and J. M. Gordillo. Phys. Rev. Lett., 87, 274501, 2001

[20] B. Selva, J. Marchalot, M.-C. Jullien. Journal of Micromechanics and Microengineering, 19, 065002, 2009.

[21] V. Miralles, A. Huerre, F. Malloggi, M.-C. Jullien. MDPI Diagnostics, 3, 33-67, 2013.

[22] B. Selva, I. Cantat, M.-C. Jullien. Physics of Fluids, 23, 052002, 2011.

[23] A. van der Net, L. Blondel, A. Saugey and W. Drenckhan. Colloids and surfaces A, 309, 159-176, 2007.

[24] J. Marchalot, J. Lambert, I. Cantat, P. Tabeling and M.-C. Jullien. $E P L, \mathbf{8 3}, 141-149,2008$.

[25] C. Gay, P. Rognon, D. Reinelt, and F. Molino. Eur. Phys. J. E, 34, 1-11, 2011

[26] S. A. Koehler, S. Hilgenfeldt and H.A. Stone. Langmuir, 16, 6327-6341 (2000).

[27] G.-F. Wang and X.-Q. Feng. Appl. Phys. Lett., 90, 231904, 2007.

[28] B. Scheid, J. Delacotte, B. Dollet, E. Rio, F. Restagno, E. A. Van Nierop, I. Cantat, D. Langevin and H. A. Stone. EPL, 90, 24002, 2010.

[1] C.-C. Wang, K.-C. Yang, K.-H. Lin, H.-C. Liu and F.-H. Lin, Biomaterials 32, 7118, 2011. 